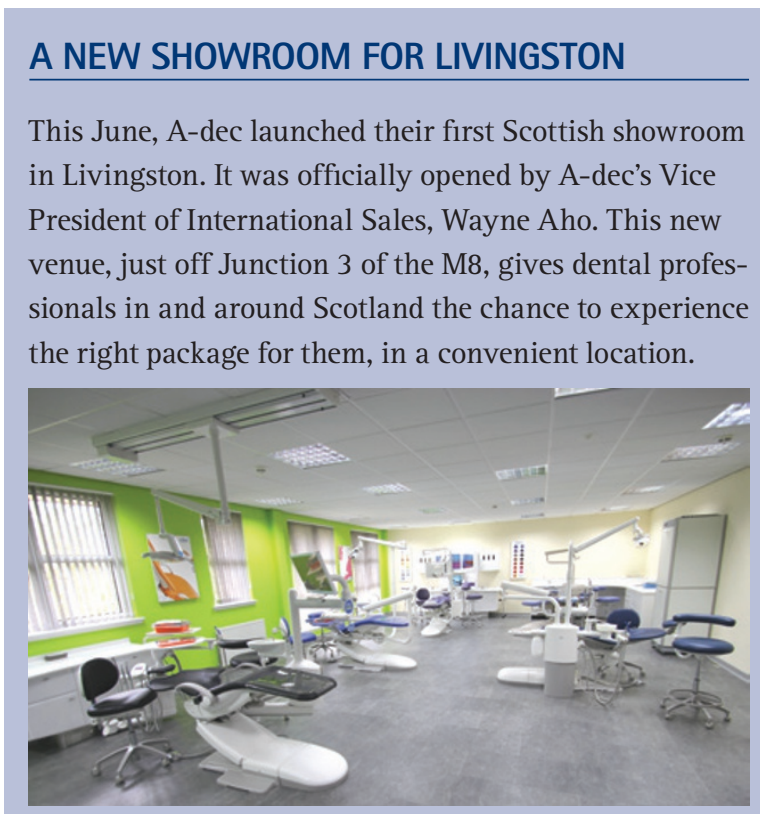

The new addition joins the three industry leading A-dec showrooms located across the UK in Surrey, Warwickshire and Manchester. Each of the experience centres contain a comprehensive range of chair packages enabling you to find a solution that works for you. Appointments to view are entirely at your convenience and individual or team member visits can be offered in the private, modern and relaxing showroom environments.

The A-dec Livingston showroom also offers a unique training venue for those looking to host dental courses in a dental environment. Whether it's a practical hands-on course or traditional lecture it is the perfect venue to host dental courses. It includes lecture style seating which comfortably seats 35 delegates, six fully loaded A-dec dental chairs for hands on practical sessions, free WiFi and AV facilities. There is even a fully functioning chair including air, water and vacuum where live dentistry can take place under the camera for the delegates to watch from their seats.

To arrange a visit to one of the four A-dec UK showrooms and test drive your ideal surgery set up call 0800233285.

www.a-dec.co.uk

\section{CONTEMPORARY TOOTH WEAR MANAGEMENT}

$\mathrm{GC}$ is the main sponsor of the Academy of Dental Excellence's course 'Contemporary tooth wear management in general practice'. This hands-on approach explains how to manage and treat tooth wear in general practice.

Taking place at LonDEC in London and presented by King's College Senior Clinical Teach, Postgraduate Lecturer and private practitioner Dr Subir Banerji, along with Dr Shamir Mehta and Mr Bill Sharpling, the course will cover tooth wear relevant to general practice in a Blended Learning format. The course speakers have extensive experience on the teaching of tooth wear at a Masters level, and have published extensively in peer reviewed journals.

The Blended Learning includes online training material accessible over five months supplemented by a two-day intensive hands-on practical techniques component held on London on Friday 5 and Saturday 6 September 2014.

An array of techniques will be taught with an emphasis on minimal intervention using additive adhesive restorations which allow for contingency planning. This comprehensive course covers both theory and practical working with high quality GC products.

For further information contact GC UK Ltd on 01908218999.

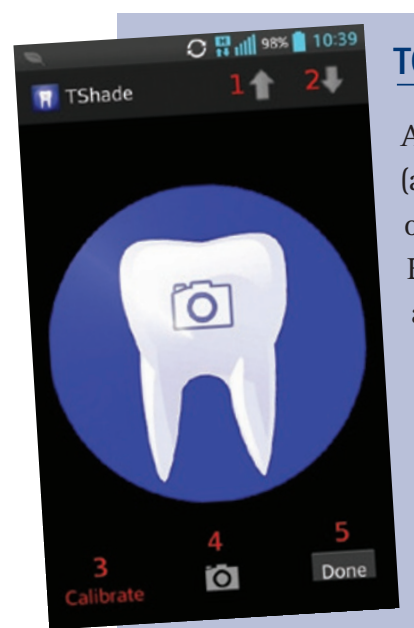

\title{
TOOTH SHADE MATCHING APP
}

Application (app) developer Stefano Bravi has created an assistant app for smartphones called TShade which is designed for matching the shades of teeth with sample tabs.

TShade is easy to use, functional and inexpensive and is expected to be of particular use within orthodontics.

To find out more, visit http:// tshade.weebly.com or purchase the app at https://play.google. com/store/apps/details?id=it. bravistefano.tshade.

\section{EASY IMPRESSIONS OF THE HIGHEST QUALITY}

Based on GC's extensive knowledge of alginate materials, GC Aroma Fine Plus has been developed to offer users final impressions of the highest quality combined with an easier and faster mixing.

With an advanced formulation, it's easy to incorporate the water into Aroma Fine Plus powder in just three seconds and the dust free formulation allows fast and vigorous mixing for a smooth bubble-free mix. Being truly thixotropic, Aroma Fine Plus reduces patient gagging, as it stays in place without dripping or running down the patient's throat during the setting phase. At the same time it flows under pressure into the narrowest of areas capturing the highest detail.

Being laboratory optimised, Aroma Fine Plus is compatible with a wide range of type IV dental stones for optimised detail reproduction and an incredibly smooth model surface.

This spearmint flavoured alginate is available in normal or fast set for personal preference. The properties of GC Aroma

Fine Plus all add up to an alginate impression material that makes it quicker and easier to take exceptionally accurate impressions. For further information please contact GC UK on 01908218999.

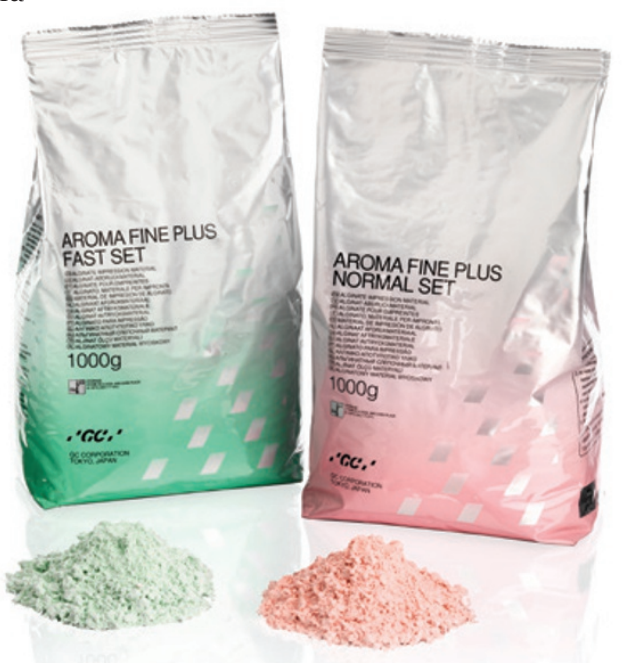

